\title{
Inhibitory simple partial (non-convulsive) status epilepticus after intracranial surgery
}

\author{
Carmel Armon, Rodney A Radtke, Allan H Friedman
}

\begin{abstract}
Objectives-To report on five patients who developed, 2 to 4 days after an intracranial neurosurgical procedure, new, persistent, focal neurological deficits which were due to inhibitory simple partial (nonconvulsive) status epilepticus, and resolved with anticonvulsant treatment.

Methods-The age range of the five patients was 15-74 years. The operations were: aneurysm clipping (three patients) and resections of an oligodendroglioma and a cavernous haemangioma (one patient each). The new focal deficits were: right hemiparesis and aphasia (two patients), aphasia alone (two patients), and left hemiparesis (one patient). The deficits were not explained by CT (obtained in all patients) or cerebral angiography (performed in two).

Results-Electroencephalography
\end{abstract}

showed, in all patients, continuous or intermittent focal seizures arising from cortex regionally relevant to the clinical dysfunction. Subtle positive epileptic phenomena (jerking) occurred intermittently in three patients as a late concommitant. Administration of anticonvulsant drugs resulted in significant improvement within 24 hours in four patients, with parallel resolution of ictal EEG activity. The fifth patient improved more slowly. Two patients relapsed when anticonvulsant concentrations fell, and improved again when they were raised.

Conclusions-It is suggested that inhibitory simple partial (non-convulsive) status epilepticus be considered in the differential diagnosis when a new unexplained neurological deficit develops after an intracranial neurosurgical procedure. An EEG may help to diagnose this condition, leading to definitive treatment. (F Neurol Neurosurg Psychiatry 2000;69:18-24)

Keywords: postoperative inhibitory seizure; ictal paralysis; ictal aphasia

Division of

Neurosurgery

A H Friedman

Correspondence to: Dr Carmel Armon, Department of Neurology, Loma Linda University, Coleman Pavillion, Room 11108, 11175 Campus Street, Loma Linda, California 92354, USA armon@discover.net

Received 2 March 1998 and in revised form

8 November 1999

Accepted 19 January 2000

inhibitory phenomena are well recogn manifestations of primary and secondarily generalised seizures, and include the "absence" and atonic manifestations of some forms of primary generalised epilepsy and the loss of consciousness which occurs in generalised tonic-clonic seizures. However, inhibitory simple partial seizures presenting as focal neurological deficits are not readily recognised. The most often recognised focal inhibitory phenomenon associated with epilepsy is postictal inhibition such as is seen with Todd's paralysis. ${ }^{1}$ This focal cortical inhibition follows a focal or secondarily generalised seizure and may last for several hours. The deficit is usually preceded by positive ictal activity, such as tonic or clonic movements. By contrast, focal impairment of neurological function as part of the ictus of a simple partial seizure is appreciated less often, although it has been recognised since $1885 .^{2-5}$

The diagnosis of persistent, inhibitory, partial simple seizures, or inhibitory partial simple (non-convulsive) status epilepticus, presenting as an isolated aphasia, or hemiparesis, or both, is often difficult to establish. ${ }^{6-12}$ In the absence of more easily recognised seizure phenomena such as jerking, epileptic seizures are not commonly considered as an aetiology of the focal deficit. To establish the diagnosis of a single inhibitory simple partial seizure, the patient must have a focal electrographic seizure that corresponds temporally and spacially with the patient's deficit; improvement in the patient's EEG must be accompanied by clinical improvement. However, when recurrent or persistent EEG seizures are associated with focal neurological deficit (inhibitory simple partial status epilepticus), it may be harder to recognise a causal role for the EEG seizures in producing the deficit. If inhibition of function due to recurrent or persistent seizures is followed by postictal inhibition (analogous to Todd's paralysis) then clinical improvement may lag behind the electrographic improvement, and its time course may be measured in hours or days, rather than seconds. Nevertheless, an epileptic aetiology of the deficit may be supported by: (a) occasionally interspersed positive epileptic phenomena (for example, jerking of paretic extremities or adjacent structures); (b) recording unequivocal onset and offset patterns of EEG seizures by extending the duration of EEG monitoring; (c) temporally linked EEG and clinical improvement associated with the administration of anticonvulsant drugs; and (d) relapses associated with low anticonvulsant concentrations, resolving when the concentrations are increased.

We report on five patients who developed a new focal deficit during the course of recovery from an intracranial neurosurgical procedure that was judged to be the result of persistent focal seizures. Each patient had focal seizures documented on EEG, and improved clinically after treatment with anticonvulsant drugs.
Patients and methods

PATIENT SELECTION

Inclusion criteria

Patients referred to the EEG laboratory at Duke University Medical Center over a 6 month period because of a new, persistent focal neurological deficit which had developed 
several days after an intracranial neurosurgical procedure were included if: (1) electrographic seizures were present in an anatomical location which could account for the neurological deficit; (2) anticonvulsant medications eliminated the EEG seizures and clinical recovery followed the resolution of EEG seizures; and (3) there was no other explanation for the neurological deficit.

\section{Exclusion criteria}

Several other patients had similar clinical histories but were excluded because: (1) global inhibitory symptoms (variable responsiveness, obtundation) predominated at the initial presentation, making the assessment of focal deficits less reliable; (2) there was underlying pathology (such as a stroke) that could account for the deficit, no clear worsening was associated with focal EEG seizures, and no clear improvement with their remission; or (3) most of the seizures were not inhibitory.

Thus we did not encounter patients with EEG seizures and focal deficits in an appropriate distribution, in the absence of an underlying structural abnormality which could account for the deficit, in whom the deficits did not improve with treatment of the seizures.

Clinical evaluation

All patients were examined by at least one of us when they were most symptomatic. Serial neurological assessments of the patients were recorded routinely by the neurosurgeons and neurologists caring for them.

CLINICAL NEUROPHYSIOLOGICALAL STUDIES

All EEGs were recorded using the 10-20 electrode placement system and standard montages, modified where necessary to accommodate the surgical incision. Continuous EEG monitoring was utilised in only one patient (patient 5). All EEGs were reviewed by two of us (CA, RAR). Criteria of onset, evolution, and cessation of the discharges helped in the recognition and classification of intermittent ictal activity. ${ }^{13}{ }^{14}$ However, these criteria could not be applied to continuous activity. Therefore, continuous focal rhythmic epileptiform EEG abnormalities were classified as ictal if the same morphology would have been classified as ictal if it had been transient or generalised, ${ }^{13-19}$ and if there was clinical and electrographic response to therapeutic concentrations of anticonvulsant medications.

Results

REPORTS OF CASES

Patient 1

A 34 year old right handed man underwent a right frontal craniotomy under local anaesthesia for gross total resection of an oligodendroglioma, carrying the resection to the sulcus anterior to the motor strip. Preoperatively, he had been placed on $300 \mathrm{mg} /$ day phenytoin. Mild left hand clumsiness on the first postoperative day resolved by postoperative day 2 . However, on postoperative day 3 the patient had a moderate left hemiparesis which became a dense hemiplegia over the next 3 days. Brain
CT on postoperative day 3 showed a small amount of extradural blood at the site of the craniotomy defect and a cystic area filled with isodense blood products at the resection site, with minimal mass effect, and could not account for the clinical deficit. The patient's phenytoin concentration was $21 \mu \mathrm{g} / \mathrm{ml}$; it was withheld, and declined to $12 \mu \mathrm{g} / \mathrm{ml}$. On postoperative day 6 several brief episodes of subtle facial twitching and head jerking without impairment of consciousness were noted. An EEG (fig 1) showed varying ictal and interictal patterns over the right frontotemporal and centrotemporal regions. The patient was treated with $1600 \mathrm{mg}$ carbamazepine. Twelve hours later there was minimal residual hemiparesis and the EEG showed right central periodic lateralised epileptiform discharges (PLEDs), recurring every $2-3$ seconds. The next day strength was normal except for mild left facial weakness. The EEG on postoperative day 12 showed right hemispheric polymorphic delta activity only. The patient was maintained on phenytoin, and had no further seizures in over 1 year of follow up.

\section{Patient 2}

A 15 year old right handed boy had twice sustained left frontal intracerebral haemorrhage from a cavernous angioma, had mild right hemiparesis and minimal aphasia, but no seizures. The cavernous angioma was resected under local anaesthesia, with no postoperative neurological change. Phenytoin given intraoperatively resulted in a serum concentration of $12 \mu \mathrm{g} / \mathrm{ml}$. On postoperative day 2 marked global aphasia developed and the right hemiparesis increased mildly. Brain CT showed extracranial soft tissue swelling, and air and subdural blood in the left parietal region (postoperative changes). Hyponatraemia (129 mEq/l) was identified and corrected. The deficits worsened between postoperative days 3 and 5. An EEG on postoperative day 5 (fig 2) showed left hemispheric periodic $1-2 \mathrm{~Hz}$ broad based triangular sharp waves with or without spikes. Increasing the serum phenytoin concentration to over $20 \mu \mathrm{g} / \mathrm{ml}$ and intermittent intravenous lorazepam produced no changes. On postoperative day 6 subtle rhythmic contractions of the right platysma were noted. Treatment with carbamazepine $(1600 \mathrm{mg} / 24 \mathrm{~h})$ resulted in significant improvement in speech and in right sided strength by the next day. On postoperative day 8, when anticonvulsant concentrations had declined, the patient and his mother identified recurrent, stereotypic episodes of transient expressive aphasia. Their EEG concomitant was electrographic seizures, consisting in a build up of left posterior temporoparietal rhythmic $2-3 \mathrm{~Hz}$ delta or $4-5 \mathrm{~Hz}$ theta activity. Serum anticonvulsant concentrations were increased and these episodes stopped within 24 hours. An EEG on postoperative day 15 showed improvement-namely, focal left temporoparietal slowing and infrequent interictal activity. At discharge the right hemiparesis and aphasia had improved to preoperative levels. The patient remained seizure free on carbamazepine for 6 months, but had recurrent 
secondarily generalised seizures on attempted withdrawal of the medication.

Patient 3

This 74 year old right handed woman had a giant anterior communicating artery aneurysm excised using a left sylvian inferior frontal approach. Angiography the next day showed that there was no spasm and the clip was in place. Anticonvulsant drugs were not given. On postoperative day 4 the patient was less alert, followed commands, was mute, but had no obvious weakness. Mild changes on head CT and angiography, which again showed no vasospasm, were not thought to account for the clinical deficit. An EEG showed left frontotemporal periodic epileptic discharges, recurring every 4-5 seconds, which evolved into runs of $1-2 \mathrm{~Hz}$ left anterior temporal spikes and sharp waves (interpreted as EEG seizures), without

A

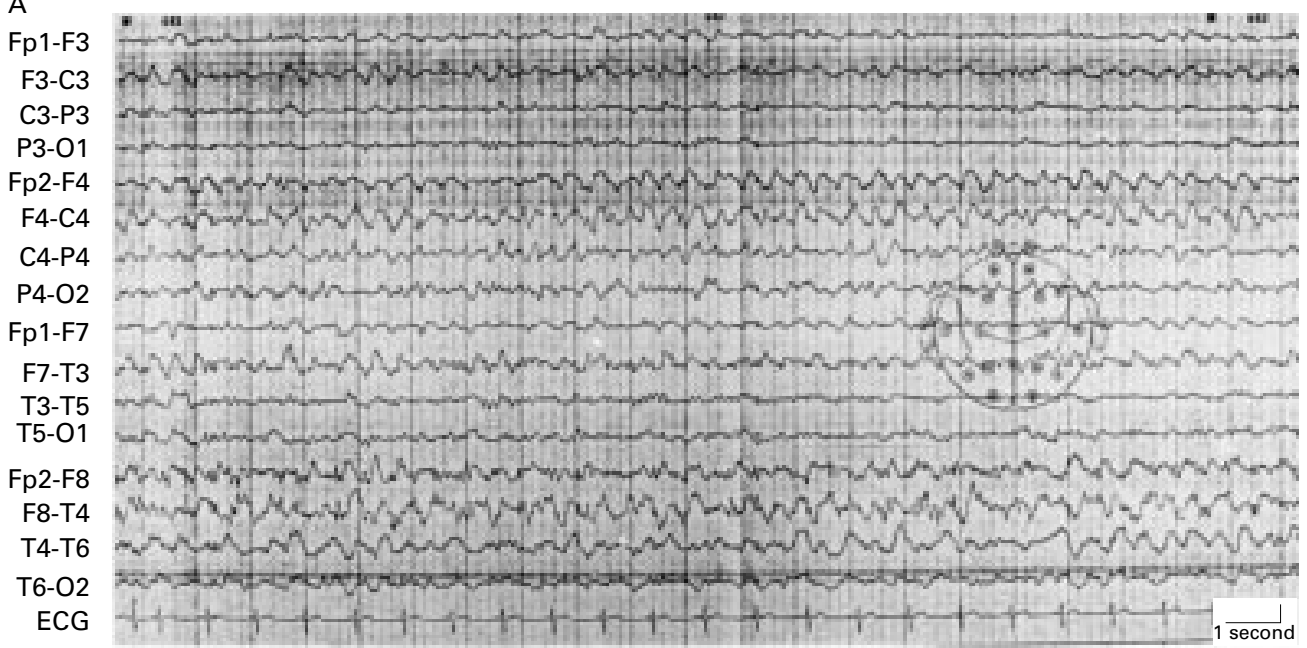

B

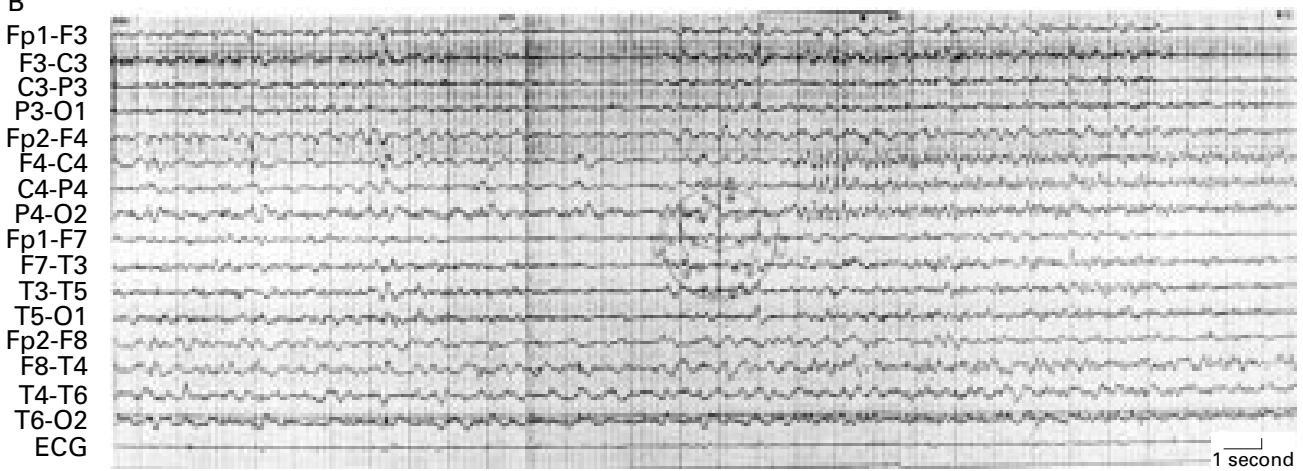

$C$

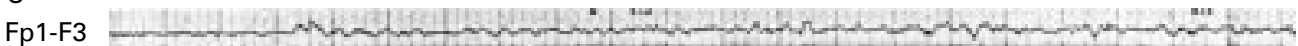

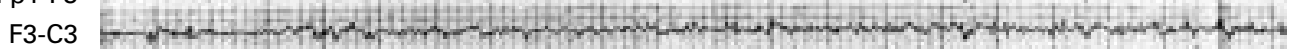
C3-P3 P3-01 Dans

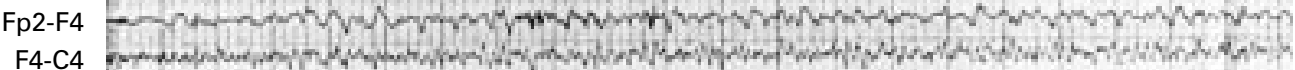

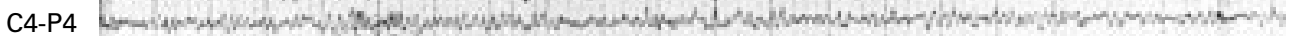

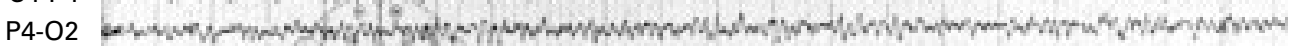

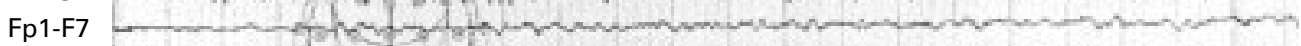

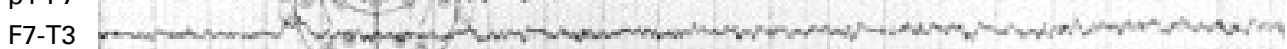

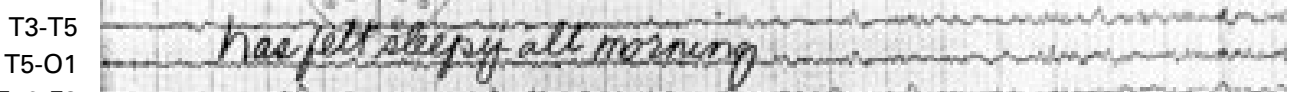

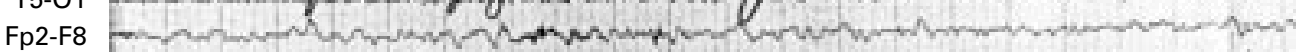

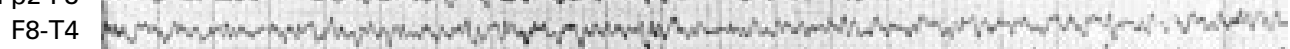

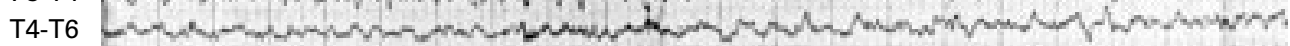

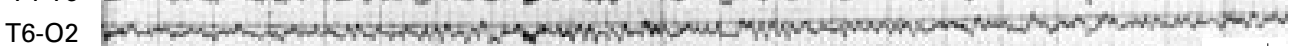
ECG

Figure 1 Patient 1, postoperative day 6. EEG shows varying ictal and interictal patterns: persistent right frontotemporal spikes and slow waves, firing at a rate of 1-3 Hz, occupied most of the recording, and, when the spikes and slow waves abated, right centrotemporal sharp waves recurring every 3-4 seconds emerged, and evolved to rhythmic right centrotemporal theta and alpha activity. Longitudinal bipolar montage. (vertical marker represents $70 \mu \mathrm{V}$ ). (A) Continuous right hemispheric rhythmic 1-3 Hz sharp and slow waves. (B and C) Brief interruption of 1-3 Hz activity, and evolution of right central rhythmic theta. 


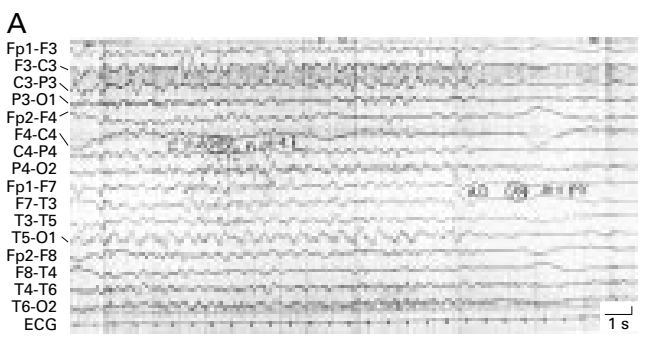

B

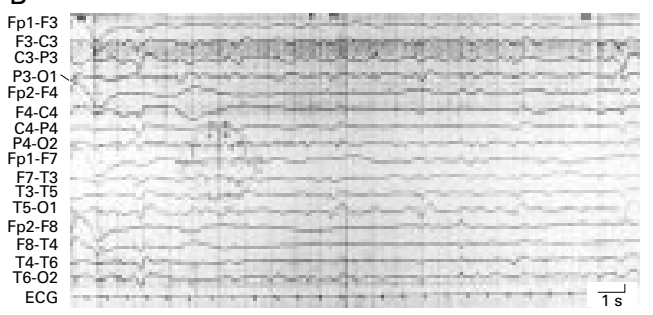

$\mathrm{C}$

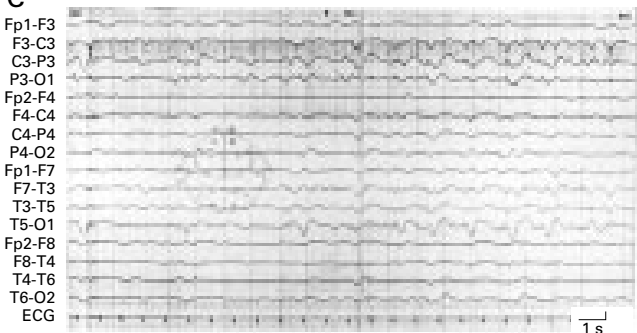

$\mathrm{D}$

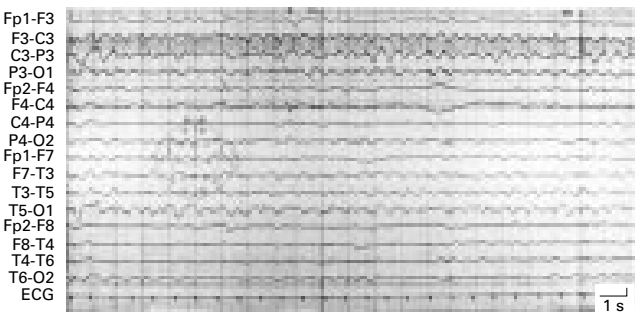

Figure 2 Patient 2, postoperative day 5. EEG shows left hemispheric periodic l-2 Hz broad based triangular sharp waves with or without spikes, during most of the recording. Longitudinal bipolar montage. (vertical marker represents $70 \mu \mathrm{V}$ ). $(A)$ Abrupt cessation of left hemispheric rhythmic delta activity. (B) (20 seconds intervening omitted) Left hemispheric PLEDs. (C and D) (20 seconds intervening omitted) Evolution of left hemispheric rhythmic delta activity.

additional behavioural changes. One electrographic seizure was more protracted and spread to the right hemisphere (as rhythmic delta) with associated worsening of the patient's responsiveness, consistent with temporary evolution to a complex partial seizure. A $2000 \mathrm{mg}$ intravenous dose of phenytoin produced a serum concentration of $11 \mu \mathrm{g} / \mathrm{ml}$. The next day the patient was more alert. The following day she was able to speak. Three days later she was alert and oriented, but "minimally confused." She had no further seizures, remained on phenytoin, and was neurologically normal during 2 years of follow up.

\section{Patient 4}

A 27 year old right handed woman underwent clip ligation of a left posterior cerebral aneurysm using a subtemporal approach. Postoperatively the patient had no neurological deficit. Aphasia occurred suddenly on postoperative day 4. Brain CT and cerebral angiography were negative. An EEG showed a non-epileptic abnormality: high voltage polymorphic delta activity over the left occipital to posterior temporal region. A loading dose of phenytoin was given. No clinical or CT changes occurred for 5 days. The EEG on postoperative day 7 showed left midtemporal sharp waves, singly and in quasiperiodic trains, evolving by postoperative day 8 to almost continuous left hemispheric PLEDs recurring every 2 seconds, maximal central-temporal. An ${ }^{18} \mathrm{~F}$-fluorodeoxyglucose positron emission tomographic (PET) scan was obtained on postoperative day 9. During radionuclide injection, EEG initially showed non-epileptic abnormalities only: left hemispheric polymorphic delta activity and no PLEDs. Nineteen minutes after the start of injection an electrographic seizure was recorded. It consisted in the evolution of rhythmic spike and wave activity at a rate of up to $2.5 \mathrm{~Hz}$ beginning in the left centroparietal and posterior temporal regions and spreading to the right temporal region, lasting for 80 seconds. Brain PET showed increased uptake in the left posterior temporalinferior parietal cortex. Treatment with carbamazepine resulted in complete resolution of the aphasia within 24 hours; EEGs on postoperative days 10 and 12 showed a non-epileptic abnormality-namely, left temporal polymorphic delta activity. Since discharge, the patient experienced a single major motor seizure when anticonvulsant concentrations were low therapeutic, but no seizures after anticonvulsant drugs were increased, and has no neurological deficit.

\section{Patient 5}

This 66 year old right handed woman underwent resection of a left paraophthalmic artery aneurysm. Her neurological examination was normal before and after surgery until postoperative day 3, when she suddenly developed right hemiparesis and aphasia. Brain CT showed a small right epidural haematoma: this did not explain the new neurological deficit. The serum phenytoin concentration was 32.6 $\mu \mathrm{g} / \mathrm{ml}$; it was held for 48 hours, and resumed at a maintenance dose after its concentration had decreased to $11 \mu \mathrm{g} / \mathrm{ml}$. An EEG on postoperative day 5 showed a non-epileptic abnormality: moderate amplitude left hemispheric polymorphic delta activity most prominently over the frontocentral and temporal regions. On postoperative day 7 the phenytoin concentration was $8.9 \mu \mathrm{g} / \mathrm{ml}$ and three episodes of rhythmic mouth activity were noted. An EEG showed left central to midtemporal spikes, which sometimes became periodic, recurring every 3 to 4 seconds. Two electrographic seizures, consisting in a build up to rhythmic left temporal 1-2 $\mathrm{Hz}$ sharp and slow waves, lasted between 1.5 and 2 minutes and resulted in no additional contemporaneous clinical change. Rhinorrhea of CSF was noted, a diagnosis of meningitis was entertained, and empirical antibiotic treat- 
ment was instituted. Brain CT showed a left sylvian subarachnoid haemorrhage, which could not account for the neurological deficit. Carbamazepine was added. Continuous EEG monitoring showed left hemispheric periodic epileptic discharges recurring every 2 to 3 seconds maximal centrotemporal. The patient gradually became more arousable on postoperative day 9 and gradually improved in responsiveness and strength. A sudden decline in her strength and alertness on postoperative day 17 , without change on CT, was attributed to reemergence of inhibitory seizures and was treated empirically by increasing the dose of phenytoin, resulting in prompt remission. She was discharged on postoperative day 19 with a mild right hemiparesis, a mild aphasia, and an attention deficit, but was doing well 6 months after discharge, ambulating alone, with no aphasia.

SUMMARY OF CLINICAL FINDINGS

Each patient awoke from an intracranial neurosurgical procedure either neurologically intact or with only a small neurological deficit. Two to 4 days after the procedure each patient developed new, severe, deficits - namely, right hemiparesis and aphasia (two patients), aphasia alone (two patients), and left hemiparesis (one patient). Radiographic investigations - at least one brain CT in all patients and a cerebral angiogram in two patients who had been operated for aneurysm - did not show a structural brain lesion or vasospasm to account for the deficit. Three patients had seizures with recognisable convulsive movements. In all three patients these clinically apparent seizures were subtle and first occurred several days after the onset of the neurological deficit. All patients had resolution of their clinical and EEG abnormalities after therapeutic concentrations of one or more anticonvulsant drugs were attained. Most patients were on phenytoin preoperatively as prophylaxis. However, one patient developed the deficit when on no anticonvulsant drugs (patient 3) and improved on therapeutic concentrations of phenytoin. The other patients, who developed their deficits when serum concentrations of phenytoin were in the therapeutic range, were treated successfully with carbamazepine. Two patients relapsed when their anticonvulsant concentrations became low. In one (patient 5), the presenting deficits recurred. In the other (patient 2), recurrent episodes of transient aphasia were recognised and confirmed electrographically to represent transient inhibitory simple partial seizures. Establishment of therapeutic serum concentrations of anticonvulsant drugs resulted in remission in both patients. After discharge, two patients (2 and 4) experienced generalised tonic-clonic seizures when their anticonvulsant concentrations became subtherapeutic.

SUMMARY OF EEG FINDINGS

Two categories of ictal EEG patterns were identified: (1) Intermittent trains of focal rhythmic alpha, theta, or delta activity (the pattern considered typical of electrographic sei- zures); and (2) continuous spikes, sharp waves, or sharp and slow wave complexes occurring at rates greater than $1 \mathrm{~Hz}$, usually at $2-3 \mathrm{~Hz}$. Both patterns have been described as ictal. ${ }^{13-18}$ The second pattern had the appearance of PLEDs, but the discharges occurred at a higher frequency than that classically described with PLEDs. Some patients had more than one ictal pattern. We did not classify periodic epileptic discharges recurring at rates under $1 \mathrm{~Hz}$ as ictal.

The percentage of recording occupied by EEG seizures at the time of persistent deficit varied. They were present almost continuously on initial EEGs in two patients (1 and 2), and occupied about $50 \%$ of the record in one patient (3). By contrast, EEG seizures were rare in two of the patients (4 and 5), and were captured only after many repeated EEG studies. The initial EEG samples in those patients showed non-epileptic abnormalitiesnamely, focal polymorphic delta activity. Epileptic changes which we interpreted as interictal (sharp waves and PLEDs at rates of less than $1 \mathrm{~Hz}$ ) developed in patient 4 before a single seizure was finally captured, and only two definite seizures were recorded in the other (patient 5). All patients had PLEDs recorded at some point in their course, yet their prognosis was better than is usually associated with PLEDs. ${ }^{20}$ Three patients had PLEDs between their EEG seizures, or on EEGs performed before definite seizures were identified. The two other patients initially had, interictally, focal sharp waves which were not periodic; PLEDs were recorded in their EEGs after the identified seizures had been treated.

\section{Discussion}

Although several reports describe transient motor paralysis secondary to focal seizures, ${ }^{2-11} 21-24$ there has been only a single report, describing three patients, in whom persistent hemiparesis was a manifestation of ongoing seizures. In all three patients the inhibitory seizures had been preceded by positive ictal phenomena. ${ }^{12}$ Three of the patients we describe had hemiparesis as part of their ictal presentation.

By contrast, it is better appreciated that inhibitory seizures may affect speech, in the form of speech arrest ${ }^{25}$ or aphasia. Speech arrest has been reported to occur from epileptic foci in either the left ${ }^{26}$ or the right ${ }^{27}$ supplementary motor area. Aphasic status epilepticus has been reported, arising from foci in the lateral cortex of the dominant hemisphere..$^{6-11}$ Inhibitory seizures have also been produced during cortical localisation studies by stimulation of the supplementary motor area or of the second somatosensory area, resulting in inhibition of motor function and speech arrest. ${ }^{28-30}$ Four of the patients we report on had aphasia as part of their ictal presentation; in two it was associated with right hemiparesis.

The five patients reported on with inhibitory simple partial (non-convulsive) status epilepticus after incracranial surgery were identified in our EEG laboratory over a period of 6 months due to a high index of suspicion. Deficits arose 
from involvement of a brain area at a distance from the surgical site. Lack of explanation of these deficits after radiological evaluation led to confirmation of their epileptic aetiology on EEG. The elimination of EEG seizures by anticonvulsant drugs was usually followed by rapid (though not immediate) resolution of the neurological deficit. Occasionally, the clinical resolution was delayed, suggesting that ictal inhibition may have been followed by varying degress of postictal inhibition. We considered alternative explanations for the deficits in these patients. The time course of onset and resolution of the deficits was incompatible with an explanation whereby reversible ischaemia was the cause of the deficits, and the EEG seizures were incidental and non-causal. The deficit often continued to develop gradually during several days after it was first identified. Yet, once treated with adequate levels of anticonvulsant drugs, it resolved over a shorter time, usually within hours or a few days. An ischaemic mechanism would be expected to result in rapid development of the deficit to its maximum, without progression, and slower resolution, independent of the use of anticonvulsant medications, and without relapse when their concentrations became low. Neuroradiological studies including CT and, in two patients angiograms, showed postoperative changes, but did not show evidence for brain oedema, ischaemia, vascular compromise, or other structural changes in the area of the deficit to a degree that would account for the deficit. However, we cannot exclude the possibility that mild, regional postoperative ischaemia lowered the seizure threshold. All patients received $10 \mathrm{mg}$ decadron every 6 hours preoperatively for 24 hours and for several days encompassing the time that the variable symptoms were recognised. Postictal (Todd's) paralysis alone cannot explain the deficits, as postictal paralysis implies recent seizures. Subtle, clinically apparent seizures (repetitive motor phenomena) were recognised only in three patients, and then only 2 to 4 days after the onset of the neurological deficit.

In two of our patients (4 and 5), definite EEG seizures were captured infrequently and only after repeated studies, and the initial EEGs showed a non-epileptic abnormalitynamely, focal polymorphic delta activity. This may have been due to failure to record infrequent EEG seizures due to limited sampling, or to lack of clear scalp representation of continuous EEG seizure activity. Possibly, focal polymorphic delta activity may have been a postictal change, the seizure presumably having occurred just before the start of the recording. This was considered in patient 4 , in whom phentoin was started in the absence of specific epileptic EEG findings. After numerous EEGs had shown no clear seizures, we obtained a PET and identified increased metabolism in the left temporoparietal cortex. This correlated well with the patient's identifed aphasia. However, a definite EEG seizure (her first recorded seizure) was obtained at the scalp 19 minutes after the start of the 20 minute radionuclide infusion, making uncertain the correlation of the PET findings with the patient's clinical state before the overt seizure. Both PET and single photon emission computed tomography (SPECT) may be useful in the diagnosis of inhibitory simple partial status epilepticus if they show increased cerebral metabolism or blood flow in an appropriate region of the brain. ${ }^{31}{ }^{32}$ Studies in the postoperative period show that such an increase does not result from surgery alone. ${ }^{33}$ However, such studies were not obtained routinely in these, or other, postoperative patients at the time. In both patients (4 and 5), adjustment of antiepileptic drug therapy, adding carbamazepine, awaited definite confirmation of the epileptic aetiology, leading to resolution of symptoms.

Whereas most of the electrographic seizures were diagnosed based on EEG changes typical of classic EEG seizures, several had continuous sharp waves or spikes as the main EEG abnormality. We considered spikes or sharp waves firing continuously at rates of $2-3 \mathrm{~Hz}$ as ictal, as have others, ${ }^{13-19}$ and treated them as seizures with anticonvulsant drugs. By contrast, we have traditionally regarded the identical wave forms firing at rates of less than $1 \mathrm{~Hz}$ as interictal PLEDs and have not treated them. This distinction may be arbitrary. There is a report of confusion in elderly people associated with PLEDs $^{34}$ resolving after treatment with anticonvulsant drugs. Our experience, as well as the limited evidence in the literature, leads us to speculate that there may be clinical significance to the rate of firing of periodic discharges, and that clinical symptoms are more likely to occur at rates above $1-2 \mathrm{~Hz}$. However, it may be equally true that all PLEDs represent inhibitory ictal phenomena, and that the rate of firing is only a quantitative feature with no special qualitative significance. A report of increased cerebral perfusion in PLEDs $^{35}$ supports this assumption. Evaluating these hypotheses will require systematic observations. Inhibitory simple partial status epilepticus may be conceptualised as the inhibitory equivalent of epilepsia partialis continua. ${ }^{36}$ The minimal residual deficits in our patients contrast with the more extensive deficits reported in animal models and humans after prolonged generalised and complex partial seizures $^{37-43}$ and may reflect the mild end of a range of dysfunction which may follow prolonged seizures.

Inhibitory simple partial (non-convulsive) status epilepticus is a treatable condition which should be suspected in any patient who, several days after an intracranial neurosurgical procedure, develops a new neurological deficit, in the absence of radiographic explanation. If focal seizures are not recorded on the first EEG, consideration should be given to attempting to document ictal patterns by increasing the sampling of EEG activity (by monitoring the EEG continuously or by obtaining additional EEG samples), or by using functional imaging studies. The recognition of inhibitory simple partial (non-convulsive) status epilepticus as the cause of the neurological deficits may lead to the institution of effective anticonvulsant treatment and to the patients' recovery. 
This work was presented in part at the 43rd annual meeting of the American Academy of Neurology, Boston, MA, USA, April 1991.

1 Todd RB. Clinical lectures on paralysis. London: Churchill, 1856.

2 Gowers WR. Epilepsia and other chronic convulsive diseases. New York: William Wood, 1885:85.

3 Jackson JH. The Lumleian lectures on convulsive seizures. BMF 1890;i:821-7.

4 Higier H. A paralytic equivalent of genuine epilepsy. Deutsche A Nervenheilk 1916;55:95-100.

5 Holmes G. Sabill memorial oration on local epilepsy. Lancet 1927;i:957-62.

6 De Pasquet EG, Gaudin ES, Bianchi A, et al. Prolonged and monosymptomatic dysphasic status epilepticus. Neurology 1976;26:244-7.

7 Hamilton NG, Matthews T. Aphasia: the sole manifestation of focal status epilepticus. Neurology 1979;29:745-8.

8 Racy A. Osborn MA, Vern BA, et al. Epileptic aphasia: first onset of prolonged monosymptomatic status epilepticus in adults. Arch Neurol 1980;37:419-22.

9 Dinner DS, Lüders H, Lederman R, et al. Aphasic status epilepticus: a case report. Neurology 1981;31:888-91.

10 Knight RT, Cooper J. Status epilepticus manifesting as Knight RT, Cooper J. Status epilepticus manifesting

11 Plesner AM, Munk-Andersen E, Luhdorf K. Epileptic aphasia and dysphoria interpreted as endogenous depresaphasia and dysphoria interpreted as en
sion. Acta Neurol Scand 1987;76:215-18.

12 Hanson PA, Chodos R. Hemiparetic seizures. Neurology 1978;28:920-3.

13 Guberman A, Couture M. Atlas of electroencephalography. Boston: Little, Brown, 1989.

14 Blume WT, Kaibara M. Atlas of adult electroencephalography. New York: Raven, 1995.

15 Treiman DM. Electroclinical features of status epilepticus. $\mathcal{F}$ Clin Neurophysiol 1995;12:343-62.

16 Treiman DM, Walton NY, Kendrick C. A progressive sequence of electroencephalographic changes during generalized convulsive status epilepticus. Epilepsy Res 1990;5: 49-60.

17 Grand'Maison F, Reiher J, Leduc CP. Restropective inventory of EEG abnormalities in partial status epileptiinventory of EEG abnormalities in partial status epilep
cus. Electroencephalogr Clin Neurophysiol 1991;264-70.

18 Granner MA, Lee SI. Nonconvulsive status epilepticus: EEG analysis in a large series. Epilepsia 1994;35:42-7.

19 Cascino GD, Westmoreland $\mathrm{BF}$, Swanson TH, et al. Seizure-associated speech arrest in elderly patients. Mayo Clin Proc 1991;66:254-8.

20 Daly DD. Epilepsy and syncope. In: Daly DD, Pedley TA, eds. Current practice of clinical electroencephalography. 2 nd ed New York: Raven, 1990:289

21 Fisher CM. Transient paralytic attacks of obscure nature: the question of non-convulsive seizure paralysis. Le fournal Canadien Des Sciences Neurologiques. 1978;5:267-73.

22 Globus M, Lavi E, Fich A, et al. Ictal hemiparesis. Eur Neurol 1982;21:165-8.

23 Lee H, Lerner A. Transient inhibitory seizures mimicking crescendo TIAs. Neurology 1990;40:165-6.
24 Kofman O, Tasker R. Ipsilateral and focal inhibitory seizures. Neurology 1967;17:1082-6.

25 Gilmore RL, Heilman KM. Speech arrest in partial seizures: evidence of an associated language disorder. Neurology 1981;31:1016-19.

26 Peled R, Harnes B, Borovich B, et al. Speech arrest and supplementary motor area seizures. Neurology 1984;34:11011.

27 Caplan LR, Zervas NT. Speech arrest in a dextral with a right mesial frontal astrocytoma. Arch Neurol 1978;35:252-

28 Penfield W, Rasmussen TB. Cerebral cortex of man. New York: MacMillan, 1950:220-1.

29 Penfield W, Jasper H. Epilepsy and the functional anatomy of the human brain. Little, Brown, 1954.

30 Lüders H, Lesser RP, Dinner DS, et al. The second sensory area in humans: evoked potential and electrical stimuation studies. Ann Neurol 1985:17:177-84.

31 Engel J Jr, Kuhl DE, Phelps ME. Regional brain metabolism during seizures in humans. Adv Neurol 1983;34:141-8.

32 Franck G, Sadzot B, Salmon E, et al. Regional cerebral blood flow and metabolic rates in human focal epilepsy and status epilepticus. Adv Neurol 1986;44:935-48.

33 Glantz MJ, Hoffman JH, Coleman E, et al. Identification of early recurrence of primary cerebral nervous system tumors by ${ }^{18} \mathrm{~F}$-fluorodeoxyglucose positron emission tomography. Ann Neurol 1991;29:347-55.

34 Terzano MG, Parrino L, Mazzucchi A, et al. Confusional states with periodic lateralized epileptiform discharges (PLEDs): a peculiar epileptic syndrome in the elderly. Epilepsia 1986;27:446-57.

35 Lee BI, Schauwecker DS. Regional cerebral perfusion in PLEDs: a case report. Epilepsia 1998;29:607-11.

36 Thomas JE, Reagan TJ, Klass DW. Epilepsia partialis continua. A review of 32 cases. Arch Neurol 1977;34:26675 .

37 Meldrum BS, Vigouroux RA, Brierly JB. Systemic factors and epileptic brain damage. Prolonged seizures in paraand epileptic brain damage. Prolonged seizures in paralyzed,

38 Söderfeldt B, Kalimo H, Olsson Y, et al. Histopathological changes in the rat brain during bicucullin-induced status epilepticus. Adv Neurol 1983;34:169-75.

39 Meldrum BS. Metabolic factors during prolonged seizures and their relation to nerve cell death. Adv Neurol 1983;34: 261-75.

40 Treiman DM, Delgado-Escueta AV. Complex partial status epilepticus. Adv Neurol 1983;34:69-81.

41 Engel J Jr, Ludwig BE, Fettell M. Prolonged partial complex status epilepticus: EEG and behavioral observations. Neurology 1978;28:863-9.

42 Treiman DM, Delgado-Escueta AV, Clark MA. Impairment of memory following prolonged complex partial status epilepticus [abstract]. Neurology 1981;31:109.

43 Krumholz A, Sung GY, Fisher RS, et al. Complex partial status epilepticus accompanied by serious morbidity and mortality. Neurology 1995;45:1499-504. 